\title{
Continuing care unit and average duration of rehabilitation in Algarve: Users perspectives
}

\begin{abstract}
Present article Refers to a study in Algarve Under, intended to understand user's perspective about care in stay. A lso describe reasons leading to satisfaction / dissatisfaction score and distinguishes significant experiences resulting from inhospital rehabilitation and Future Needs after discharge. This qualitative study supports on five open questions for semi-structured interview. The sample is composed by 33 users, mostly female, aged 33 to 70 years, married and living with partner. Users mentioned lack of family support, extended loss of autonomy and physical capacity, effectiveness in health care and families monitoring care the main restriction contribution for satisfaction / dissatisfaction score. Positive feelings about recovery, longer period of rehabilitation and fear of permanent disabilities were the most significant experiences identified. in home return informal caregivers support is the most pointed needs.
\end{abstract}

Volume 2 Issue 5 - 2017

\author{
Antonio Duarte \\ Coordinator of the Department of Occupational Therapy, \\ Hospital Center of Algarve, Portugal
}
Correspondence: Antonio Duarte, Licensed in Occupational Therapy Coordinator of the Department of Occupational Therapy Hospital Center of Algarve, Portugal, Email ajaduarte@gmail.com

Received: August 19,2017| Published: December 01, 2017

Keywords: uccmdr, relocation, addiction, satisfaction, experiences, needs

\section{Introduction}

We have tried to meet the significant experiences on resumption of independence resulting from the relocation of medium duration units and rehabilitation and future needs in the home. The evaluation of user satisfaction indicators, such as the professional performance and communication and relationship with the professionals is an essential aspect for the quality of services. ${ }^{1}$ Some studies show que these users face various difficulties and needs in preparation of your home, such as solid social support network BOTH formal, the informal ${ }^{2}$ To provide physical help on functional incapacity and loss of autonomy ${ }^{3}$ Pandey \& Malik et al. ${ }^{4}$ as well as emotional support. Pass to independent dependent generates fear and uncertainty when the social reintegration. Experiences that allow you to identify needs in the home. ${ }^{4}$

The "need" formal support networks (healthcare professionals) or informal support (family and persons significant) is assumed by users [2], the Latter in the essential tasks, physical help on functional incapacity is assumed and the loss of autonomy. 3,5

However, there are people without caregivers. Live alone with loss or dysfunction of family ties. The need for learning new skills at home, assuming recast on achievement and compensation in the tasks resulting from the malfunction (S 2007).

In this context, the main objective of this study was to understand the meaning attributed to perspectives of users admitted to UCCMDR, Specifically meet relocation Reasons, reasons of (in) satisfaction, significant experiences on resumption of independence and future needs in the home.

\section{Method}

Qualitative methodology was ADOPTED, supported in the meanings of the participants for your experiential flexibility, detail and emergency ${ }^{6}$ Remondes CJ \& Parents R et al. 2012. The 33 participants for convenience in UCCMDR, showed cognitive ability to dialogue and allowed to record the interview. Were predominantly female
$(60 \%)$, aged $33-70$ years $($ mean $=63.8 \%)$, married $(52 \%)$, living with the spouse $(60 \%)$ children / grandchildren $(32 \%)$ or alone $(8 \%)$.

\section{Procedure}

The collection was made between 15 February and 30 March 2015 in UCCMDR, after informed consent of participants According to the Helsinki Protocol. ${ }^{7}$

\section{Results}

\section{Internment}

Users $(n=22)$, consider the lack of family Support / people and the loss of autonomy and physical capacity, the Reasons for the relocation, the absence or scarcity of family / support persons significant, compounded by chronic illness without in the autonomy activities of daily Living (ADSL).

\section{Satisfaction with relocation}

The treatment is considered by users $(n=28)$ is the cause satisfaction. Communication and relationship with the professionals, are Considered grounds for satisfaction by users $(n=30)$, translated into confidence, availability and affectivity. The relationship Involves a communication to help overcome the dependency for the trust promoters environments be Heard, is considered essential for the person to feel the presence of professionals, like help available. The admission Enables, that an equal number of Clients $(n=33)$ Consider the food and hygiene the reason for satisfaction on the organization and operation of the unit.

\section{Dissatisfaction with the internment}

It is evidenced in some users $(n=16)$, dissatisfaction with the continuity of care by restriction of relatives / persons significant in your follow-up. It is desirable that, in Accordance with the situation of the wearer and the constraints of the service, if you integrate the team as a partner in care, a significant person / family, aiming at ensuring your continued after discharge. 


\section{Resumption resulting of independence from the relocation}

The recovery of independence resulting from Relocation is size that users have difficulty thinking. However, most $(n=27)$ Referred to positive feelings of improvement, Despite the chronicity of addiction. The need for more time for the functional recovery of independence resulting from relocation is significant experience for users $(n=15)$, associated with the fear of remain of limitations, to others. The functionality is a critical faculty. Obtaining from date the functional and social spheres becomes precious, is being disease indicator, internment, factor of likelihood of functional Recovery in Rehab programs, and professional therapeutic goal is independence. Home All the users $(n=33)$ considers to have needs in the home. For $(n=24)$, informal require caregivers, and to a lesser number of formal. The change in the family instrument is functioning need considered by the minority $(\mathrm{n}=9)$.

\section{Discussion and conclusion}

This study had the main objective to understand the meaning of the perspectives of users admitted to UCCMDR, Specifically the meeting Reasons for relocation and the reasons that Contribute to the (in) same satisfaction. We have tried to meet the significant experiences on resumption of independence resulting from relocation and future requirements.

With the proximity of the high, it is important to understand the concerns of users in home. Family / significant people represent the key resource on Home maintenance of dependent person, and the effectiveness of informal care requires the presence of support networks que provide resources in response to the demands.

Homes, home support services, day centers and rehabilitation Institutions, in the "formality" of care represent added value in cuidativa continuity. Users require so, to learn new skills that will enable Them to face the tasks inherent to the home, to feel capable and motivated to Respond to future challenges. Users who refer the amendment to the family the instrumental functioning future need, think about Changes in family dynamics and Appropriate to your residential building functional capacity, at home. The daily take on greater Instrumental Activities significance in the case of a dependent family who need help. ${ }^{8,9}$

\section{Acknowledgements}

None.

\section{Conflict of interest}

Authors declare that there are no conflicts of interest.

\section{References}

1. Pereira G, Araujo Soares V, McIntyre T. User satisfaction and attitudes towards doctors and medicine: A pilot study. Psychology Health \& Diseases. 2001;2(2):69-80.

2. Pereira M, Roncon J. Family relationship in the elderly: Adaptation of the Family Relations Index (IFR). Psychology Health \& Diseases. 2010;11(1):41-53.

3. Figueiredo H. Dynamic model of evaluation and family intervention: A collaborative approach d and family nursing. Loures: Luso science. $2012 ; 63$

4. Schumacher L, Beidler M, Beeber S, et al. The transactional model of cancer family caregiving skill. ANS Adv Nurs Sci. 2006;29(3):271-286.

5. Peixoto J, Martins T. Profile adaptation $r$ esiliência familiar to the Portuguese population. Psychology, Health \& Diseases. 2012;13(2):372-388

6. Relinque S, Arroyo G, Fernande G. Tips PRACTICAL for escribir un artículo qualitative published en Psychology. Psychosocial Intervention. 2013;22(1):71-79.

7. Di Ruggiero M. Declaración Helsinki, Principi the values y en bioethic game en la investigación medical con humans. Colombian Journal of Bioethics. 2011;6(1):125-144.

8. Pedro L, Ribeiro PJ. Psycho metric analysis of the scale of impact on autonomy and participation in people with multiple sclerosis. Psychology, Health \& Diseases. 2008;9(2):271-281.

9. Petronilho S. Preparation of the homecoming. Coimbra: Formasau, Portugal; 2007. 